IMA Journal of Applied Mathematics (2014) 1-20

doi: 10.1093/imamat/dri017

\title{
A Finite Element Approach to Modelling Fractal Ultrasonic Transducers
}

\author{
Ebrahem A. Algehyne And Anthony J. Mulholland \\ Department of Mathematics and Statistics, University of Strathclyde, \\ 26 Richmond Street, Glasgow, G1 1XH, UK.
}

\begin{abstract}
[Received on 22 April 2014]
Piezoelectric ultrasonic transducers usually employ composite structures to improve their transmission and reception sensitivities. The geometry of the composite is regular with one dominant length scale and, since these are resonant devices, this dictates the central operating frequency of the device. In order to construct a wide bandwidth device it would seem natural therefore to utilize resonators that span a range of length scales. In this article we derive a mathematical model to predict the dynamics of a fractal ultrasound transducer; the fractal in this case being the Sierpinski gasket. Expressions for the electrical and mechanical fields that are contained within this structure are expressed in terms of a finite element basis. The propagation of an ultrasonic wave in this transducer is then analyzed and used to derive expressions for the non-dimensionalised electrical impedance and the transmission and reception sensitivities as a function of the driving frequency. Comparing these key performance measures to an equivalent standard (Euclidean) design shows some benefits of these fractal designs.
\end{abstract}

Keywords: finite element method, fractal, ultrasound, transducer, renormalisation

\section{Introduction}

Ultrasonic transducers are devices that convert electrical energy into mechanical vibration and conversely can convert mechanical energy into an electrical signal ( Yang (2006); Hayward et al. (1984); Mulholland \& Walker (2011)). These devices can be used to interrogate a medium by emitting a wave (electrical to mechanical) and then listening to the same wave after it has traversed the medium (mechanical to electrical). Piezoelectric ultrasonic transducers typically employ composite structures to improve their transmission and reception sensitivities ( Hayward (1984); Orr et al. (2007)) and many biological species produce and receive ultrasound such as moths, bats, dolphins and cockroaches. The manmade transducers tend to have very regular geometry on a single scale whereas the natural systems exhibit a wide variety of intricate geometries often with resonators over a range of length scales ( Müller et al. (2006); Müller (2004); Miles \& Hoy (2006); Chiselev et al. (2009); Eberl et al. (2000); Nadrowski et al. (2008); Robert \& Göpfert (2002); Montero de Espinosa et al. (2005)). This allows these transducers to operate over a wider frequency range and hence results in reception and transmission sensitivities with exceptional bandwidths. To assess the benefits of having transducers with such structures it would be useful to build mathematical models of them. One structure whose geometrical components consist of a range of length scales is a fractal. There have been a number of mathematical approaches which describe wave propagation in fractal media ( Kigami (2001); Falconer (2003); Giona (1996); Abdulbake \& Mulholland (2003); Abdulbake et al. (2004)). This paper constructs a model of a fractal ultrasound transducer and then uses this model to compare its operational qualities with that of a standard (Euclidean) design. Previously this topic was examined using a finite differences approach ( Mulholland \& Walker (2011)). Previously this topic was examined using a finite differences approach ( Mulholland \& Walker (2011)) whereby each edge in the fractal lattice was modelled as a one dimensional piezoelectric 
bar whose only degree of freedom was in the plane of the lattice. This model did not therefore allow for other types of motion of the lattice, or directions of the electric field, and was essentially a local description of the dynamics of each edge that when joined to the other edges to form the lattice, led to the global dynamics of the device. This paper will derive the governing equations from the general tensor equations for the whole lattice so that the three dimensional world that the device is embedded within is accounted for. This framework allows different parameterisations to be deployed and in this paper we will examine the case where the displacement acts out of the plane of the lattice whereas the electric field operates within the plane of the lattice. This will allow us to consider the transverse modes of the device. In addition, this paper will be the first to use a finite element methodology as the basis for the renormalisation approach and it is precisely this global approach to modelling the device that permits this type of analysis. This renormalisation approach will be used in this paper to derive expressions for the key operational characteristics of the device. Of course, from a manufacturing respective only the pre-fractal (finite fractal generation level) gaskets can be feasibly constructed and so an investigation into the dependency of the device characteristics at a low fractal generation level is undertaken here. The fractal that will be used in this article to simulate this self-similar transducer is the Sierpinski gasket ( Falconer \& Hu (2001)). Such an ultrasonic transducer would start with an equilateral triangle of piezoelectric crystal, and the next generation $(n=1)$ would be obtained by replacing this by three copies of itself, each of which being half the size of the original triangle. This process is then repeated for several generations (see Figure 1). The Sierpinski gasket lattice of degree 3, $\mathrm{SG}^{(n)}(3)$, is the lattice counterpart of the Sierpinski gasket ( Schwalm (1988)) (see Figure 2). This lattice is constructed by a process which starts from the Sierpinski gasket of order $n=1$ (which consists of three piezoelectric triangles), assigns a vertex to the centre of each of these triangles and, by connecting these vertices together with edges, the lattice at generation level $n=1\left(\mathrm{SG}^{(1)}(3)\right)$ is constructed. The lattice has side length $L$ units which remains constant as the generation level $n$ increases. Therefore, as $n$ increases, the length of the edge between adjacent vertices tends to zero and in this limit the lattice will perfectly match the space filling properties of the original Sierpinski gasket ( Mulholland (2008)). The total number of vertices is $N=3^{n}$ and $h^{(n)}=L /\left(2^{n}-1\right)$ is the edge length of the fractal lattice. The vertex degree is 3 apart from the boundary vertices (input/output vertices) which have degree 2 and $M=3\left(3^{n}-1\right) / 2$ denotes the total number of edges. These boundary vertices will be used to interact with external loads (both electrical and mechanical) and so we introduce fictitious vertices $A, B$ and $C$ to accommodate these interfacial boundary conditions (see Figures 3 and 4 ). Let us denote by $\Omega$ the set of points lying on the edges and vertices of $\mathrm{SG}^{(n)}(3)$ and denote the region's boundary by $\partial \Omega$.

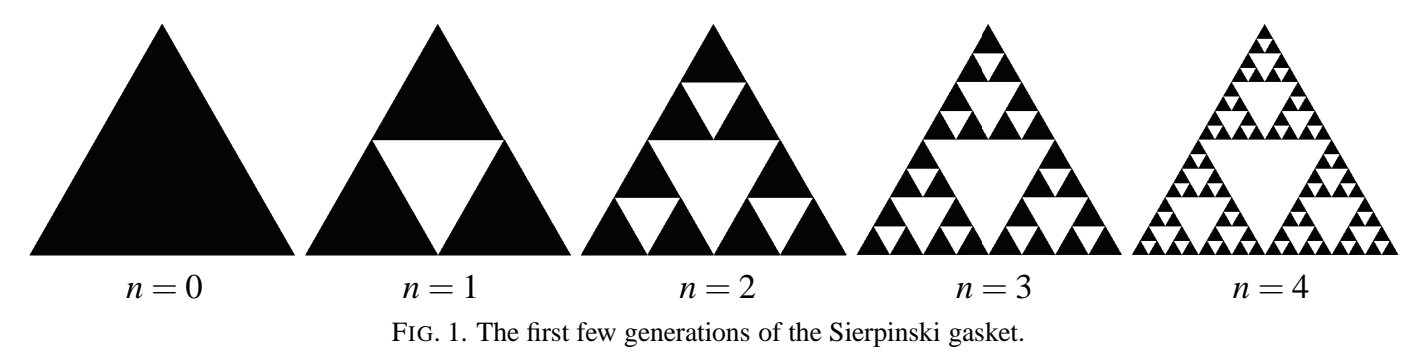




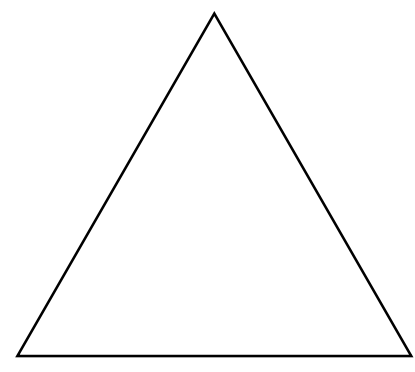

$n=1$

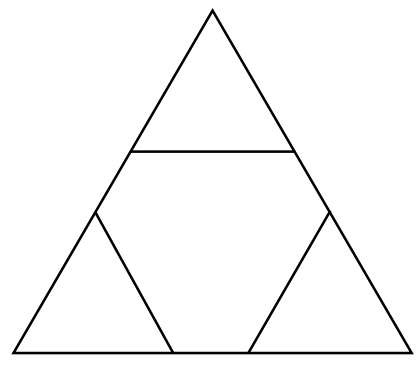

$n=2$

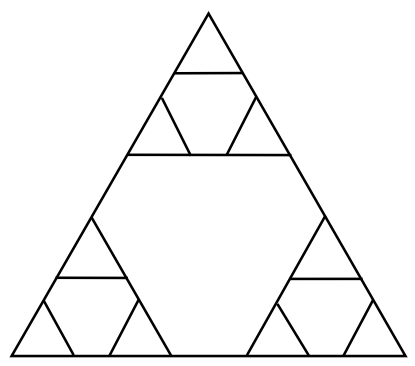

$n=3$

FIG. 2. The first few generations of the Sierpinski gasket lattice $S G(3)$.

\section{Model Derivation}

The lattice represents the vibrations of a piezoelectric material (the focus here is on PZT-5H Auld (1973)) that has been manufactured to form a Sierpinski gasket. The interplay between the electrical and mechanical behaviour of the lattice vertices is therefore described by the piezoelectric constitutive equations ( Yang (2006))

$$
\begin{aligned}
T_{i j} & =c_{i j k l} S_{k l}-e_{k i j} E_{k}, \\
D_{i} & =e_{i k l} S_{k l}+\varepsilon_{i k} E_{k},
\end{aligned}
$$

where $T_{i j}$ is the stress tensor, $c_{i j k l}$ is the stiffness tensor, $S_{k l}$ is the strain tensor, $e_{k i j}$ is the piezoelectric tensor, $D_{i}$ is the electrical displacement tensor and $\varepsilon_{i k}$ is the permittivity tensor (where the Einstein summation convention is adopted). The strain tensor is related to the displacement gradients $u_{i, j}$ by $S_{i j}=\left(u_{i, j}+u_{j, i}\right) / 2$. The dynamics of the piezoelectric material is then governed by

$$
\rho_{T} \ddot{u}_{i}=T_{j i, j}
$$

subject to Gauss' law

$$
D_{i, i}=0
$$

where $\rho_{T}$ is the density and $u_{i}$ is the component of displacement in the direction of the $i^{\text {th }}$ basis vector. So, combining equations (2.3) and (2.1) gives

$$
\rho_{T} \ddot{u}_{i}=c_{j i k l} S_{k l, j}-e_{k j i} E_{k, j},
$$

and combining equations (2.4) and (2.2) gives

$$
D_{i, i}=e_{i k l} S_{k l, i}+\varepsilon_{i k} E_{k, i}=0 .
$$

In this paper attention is restricted to the out of plane displacement only (a horizontal shear wave) by stipulating that $\underline{u}=\left(0,0, u_{3}\left(x_{1}, x_{2}, t\right)\right)$, this choice of paramerisation will simplify the algebra significantly and will lead to a scalar dynamical equation. It also will allow us to consider the transverse vibrations of the device which is of engineering interest in the application of this device. There are of course other parameterisations that could be chosen and a suitable choice would also afford the study 
of the vector elastodynamical equations. Now if $\underline{E}=\left(E_{1}\left(x_{1}, x_{2}\right), E_{2}\left(x_{1}, x_{2}\right), 0\right)$ then, for PZT-5H Auld (1973), equation (2.5) becomes

$$
\rho_{T} \ddot{u}_{3}=c_{44}\left(u_{3,11}+u_{3,22}\right)-e_{24}\left(E_{1,1}+E_{2,2}\right),
$$

where the Voigt notation has been used to express these tensors as matrices. For example, $c_{44} \equiv c_{2323}$ and $e_{24} \equiv e_{223}$. Equation (2.6) gives

$$
E_{1,1}+E_{2,2}=-\frac{e_{24}}{\varepsilon_{11}}\left(u_{3,11}+u_{3,22}\right)
$$

where $\varepsilon_{22}=\varepsilon_{11}$ for PZT-5H. Combining these two equations gives

$$
\ddot{u}_{3}=c_{T}^{2} \nabla^{2} u_{3}
$$

where $\nabla^{2}=\left(\partial^{2} / \partial x_{1}^{2}+\partial^{2} / \partial x_{2}^{2}\right), c_{T}=\sqrt{\mu_{T} / \rho_{T}}$ is the (piezoelectrically stiffened) wave velocity and $\mu_{T}=c_{44}\left(1+e_{24}^{2} /\left(\varepsilon_{11} c_{44}\right)\right)$ is the piezoelectrically stiffened shear modulus, subject to the initial conditions $u_{3}(\underline{x}, 0)=\dot{u}_{3}(\underline{x}, 0)=0$ and the boundary conditions of continuity of displacement and force at $\partial \Omega$. By introducing the non-dimensionalised variable $\theta=c_{T} t / h$, dropping the subscript on $u$, and applying the Laplace transform $\mathscr{L}: \theta \rightarrow q$ gives

$$
q^{2} u=h^{2} \nabla^{2} u
$$

Now seek a weak solution $u \in H^{1}(\Omega)$ where on the boundary $u=u_{\partial \Omega} \in H^{1}(\partial \Omega)$. Multiplying by a test function $w \in H_{B}^{1}(\Omega)$, where $H_{B}^{1}(\Omega):=\left\{w \in H^{1}(\Omega): w=0\right.$ on $\left.\partial \Omega\right\}$, integrating over the region $\Omega$, and using Green's first identity gives

$$
\int_{\Omega}\left(q^{2} u w+h^{2} \nabla u \cdot \nabla w\right) d \underline{x}=0 .
$$

\section{Galerkin discretisation}

Using a standard Galerkin method we replace $H^{1}(\Omega)$ and $H_{B}^{1}(\Omega)$ by the finite dimensional subspaces $S$ and $S_{B}=S \cap H_{B}^{1}(\Omega)$. Let $U_{B} \in S$ be a function that approximates $u_{\partial \Omega}$ on $\partial \Omega$, then the discretised problem involves finding $U \in S$ such that

$$
\int_{\Omega}\left(q^{2} U W+h^{2} \nabla U \cdot \nabla W\right) d \underline{x}=0
$$

where $W$ is the test function expressed in this finite dimensional space. Let $\left\{\phi_{1}, \phi_{2}, \cdots, \phi_{N}\right\}$ form a basis of $S_{B}$ and set $W=\phi_{j}$, then

$$
\int_{\Omega}\left(q^{2} U \phi_{j}+h^{2} \nabla U \cdot \nabla \phi_{j}\right) d \underline{x}=0 .
$$

Furthermore, let $\phi_{I}, I=\{N+1, N+2, N+3\}$ form a basis for the boundary nodes and let

$$
U=\sum_{i=1}^{N} U_{i} \phi_{i}+\sum_{i \in I} U_{B_{i}} \phi_{i}
$$

Hence, equation (3.2) can be written as

$$
A_{j i}^{(n)} U_{i}^{(n)}=b_{j}^{(n)}
$$


where

$$
\begin{gathered}
b_{j}^{(n)}=-\sum_{i \in I}\left(\int_{\Omega}\left(q^{2} \phi_{i} \phi_{j}+h^{2} \nabla \phi_{i} . \nabla \phi_{j}\right) d \underline{x}\right) U_{B_{i}}, \\
A_{j i}^{(n)}=q^{2} H_{j i}^{(n)}+h^{2} K_{j i}^{(n)}, \\
K_{j i}^{(n)}=\int_{\Omega}\left(\nabla \phi_{j} . \nabla \phi_{i}\right) d \underline{x},
\end{gathered}
$$

and

$$
H_{j i}^{(n)}=\int_{\Omega}\left(\phi_{j} \phi_{i}\right) d \underline{x} .
$$

The lattice basis function at vertex $\underline{x}_{j}$ is chosen to be

$$
\phi_{j}(x, y)= \begin{cases}a+b x+c y+d\left(x^{2}+y^{2}\right) & j \in\{1, \cdots, N\} \\ a+d\left(x^{2}+y^{2}\right) & j \in I .\end{cases}
$$

where $(x, y) \in \Omega$ and $a, b, c, d \in \mathbb{R}$ are coefficients to be determined. Futhermore, the $\phi_{j}$ are defined as localised basis functions such that

$$
\phi_{j}(x, y)= \begin{cases}1 & \text { if }(x, y)=\left(x_{j}, y_{j}\right) \\ 0 & \text { if }(x, y)=\text { coordinates of vertices adjacent to vertex } j\end{cases}
$$

and $\phi_{j}(x, y)=0$ at all points which do not lie in the edges adjacent to vertex $j$. For each generation level of the $\mathrm{SG}^{(n)}(3)$ lattice the coordinates of the vertices are known. Using equation (3.10) the coefficients in equation (3.9) can be determined. For a particular element lying between vertex $i$ and vertex $j$ the isoparametric representation, given by $(x(s), y(s))=\left(\left(x_{j}-x_{i}\right) s+x_{i},\left(y_{j}-y_{i}\right) s+y_{i}\right)$ is employed, where $s_{1}=0$ and $s_{2}=1$ and $d \underline{x}=h d s$. Substituting this into equation (3.7) gives for each element (edge) $e$ where $e=1, \cdots, M$

$$
{ }^{e} K_{j i}^{(n)}=\frac{4}{h}\left\{\begin{array}{lll}
\int_{0}^{1} s^{2} d s & =\frac{1}{3} & \text { if } j=i=p \\
\int_{0}^{1} s(s-1) d s=-\frac{1}{6} & \text { if }(j=p \text { and } i=q) \text { or }(j=q \text { and } i=p) \\
\int_{0}^{1}(s-1)^{2} d s=\frac{1}{3} & \text { if } j=i=q \\
0 & & \text { otherwise }
\end{array}\right.
$$

where element $e$ connects node $p$ to node $q$. For the boundary elements $e \in\{M+1, M+2, M+3\}$

$$
{ }^{e} K_{j i}^{(n)}= \begin{cases}\frac{4}{3 h} & \text { if } j=i=q \\ 0 & \text { otherwise }\end{cases}
$$

where $q$ is the corner node of the $S G(3)$ lattice connected to element $e$ (for $n=1, q \in\{1,2,3\}$, and for $n=2, q \in\{1,5,9\})$. Equations (3.11) and (3.12) can then be used to assemble the full matrix in equation (3.7). Similarly, for ${ }^{e} H_{j i}^{(n)}$ where $e \in\{1, \cdots, M\}$, equation (3.8) leads to

$$
{ }^{e} H_{j i}^{(n)}=h\left\{\begin{array}{lll}
\int_{0}^{1}\left(s^{2}-1\right)^{2} d s & =\frac{8}{15} & \text { if } j=i=p \\
\int_{0}^{1}\left(s^{2}-1\right)(s-2) s d s & =\frac{11}{30} & \text { if }(j=p \text { and } i=q) \text { or }(j=q \text { and } i=p) \\
\int_{0}^{1}(s-2)^{2} s^{2} d s & =\frac{8}{15} & \text { if } j=i=q \\
0 & & \text { otherwise }
\end{array}\right.
$$




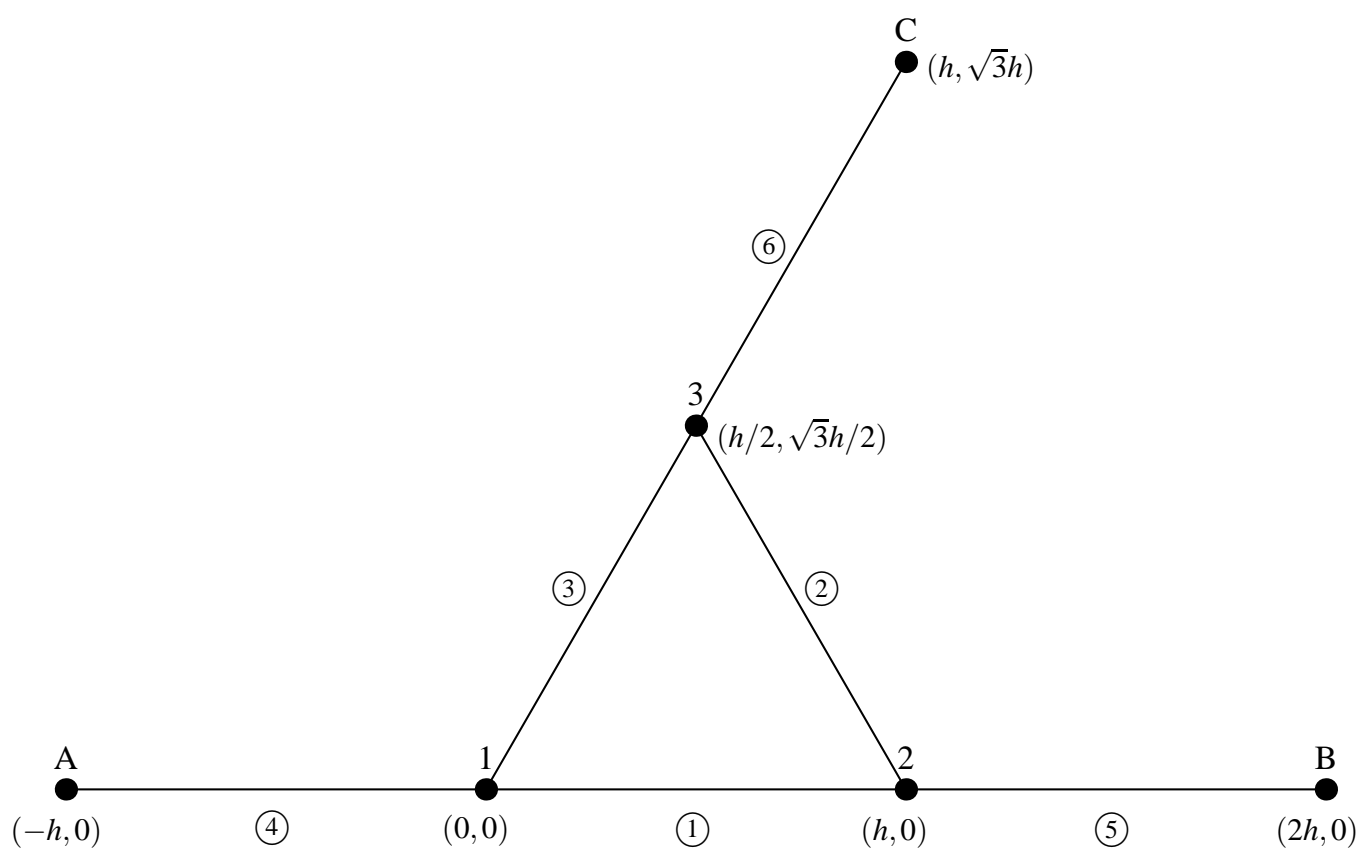

FIG. 3. The Sierpinski Gasket lattice $S G(3)$ at generation level $n=1$. Nodes 1,2 and 3 are the input/output nodes, and nodes $A, B$ and $C$ are fictitious nodes used to accommodate the boundary conditions. The lattice has 6 elements (circled numbers), with two vertices adjacent to each element.

where element $e$ connects node $p$ to node $q$, and for the boundary elements $e \in\{M+1, M+2, M+3\}$

$$
{ }^{e} H_{j i}^{(n)}=h \begin{cases}\int_{0}^{1}\left(s^{2}-1\right)^{2} d s=\frac{8}{15} & \text { if } j=i=q \\ 0 & \text { otherwise }\end{cases}
$$

where $q$ is the corner node of the $S G(3)$ lattice connected to element $e$. Combining equations (3.11),(3.12),(3.13) and (3.14) gives equation (3.6) as $A_{j i}^{(1)}=h \alpha$ if $j=i$ and $h \beta$ otherwise where $\alpha=4+\left(8 q^{2} / 5\right)$, and $\beta=(-2 / 3)+11 q^{2} / 30$. In general $A_{j i}^{(n)}=\bar{A}_{j i}^{(n-1)}+\beta V_{j i}^{(n)}$, where $\bar{A}_{j i}^{(n-1)}$ is a block diagonal matrix consisting of 3 copies of $A_{j i}^{(n-1)}$ and $V_{j i}^{(n)}$ is the adjacency matrix for the subgraph of $\mathrm{SG}^{(n)}(3)$ consisting of the edges that connect each of the three $\mathrm{SG}^{(n-1)}(3)$ graphs. A similar treatment can be given to equation (3.5) to give (where $m=(N+1) / 2$ )

$$
b_{j}^{(n)}=\left\{\begin{array}{ll}
-\left(\int_{e_{M+1}}\left(q^{2} \phi_{N+1} \phi_{j}+h^{2} \nabla \phi_{N+1} \cdot \nabla \phi_{j}\right) d \underline{x}\right) U_{A}, & j=1 \\
-\left(\int_{e_{M+2}}\left(q^{2} \phi_{N+2} \phi_{j}+h^{2} \nabla \phi_{N+2} \cdot \nabla \phi_{j}\right) d \underline{x}\right) U_{B}, & j=m \\
-\left(\int_{e_{M+3}}\left(q^{2} \phi_{N+3} \phi_{j}+h^{2} \nabla \phi_{N+3} \cdot \nabla \phi_{j}\right) d \underline{x}\right) U_{C}, & j=N \\
0 & \text { otherwise }
\end{array} .\right.
$$




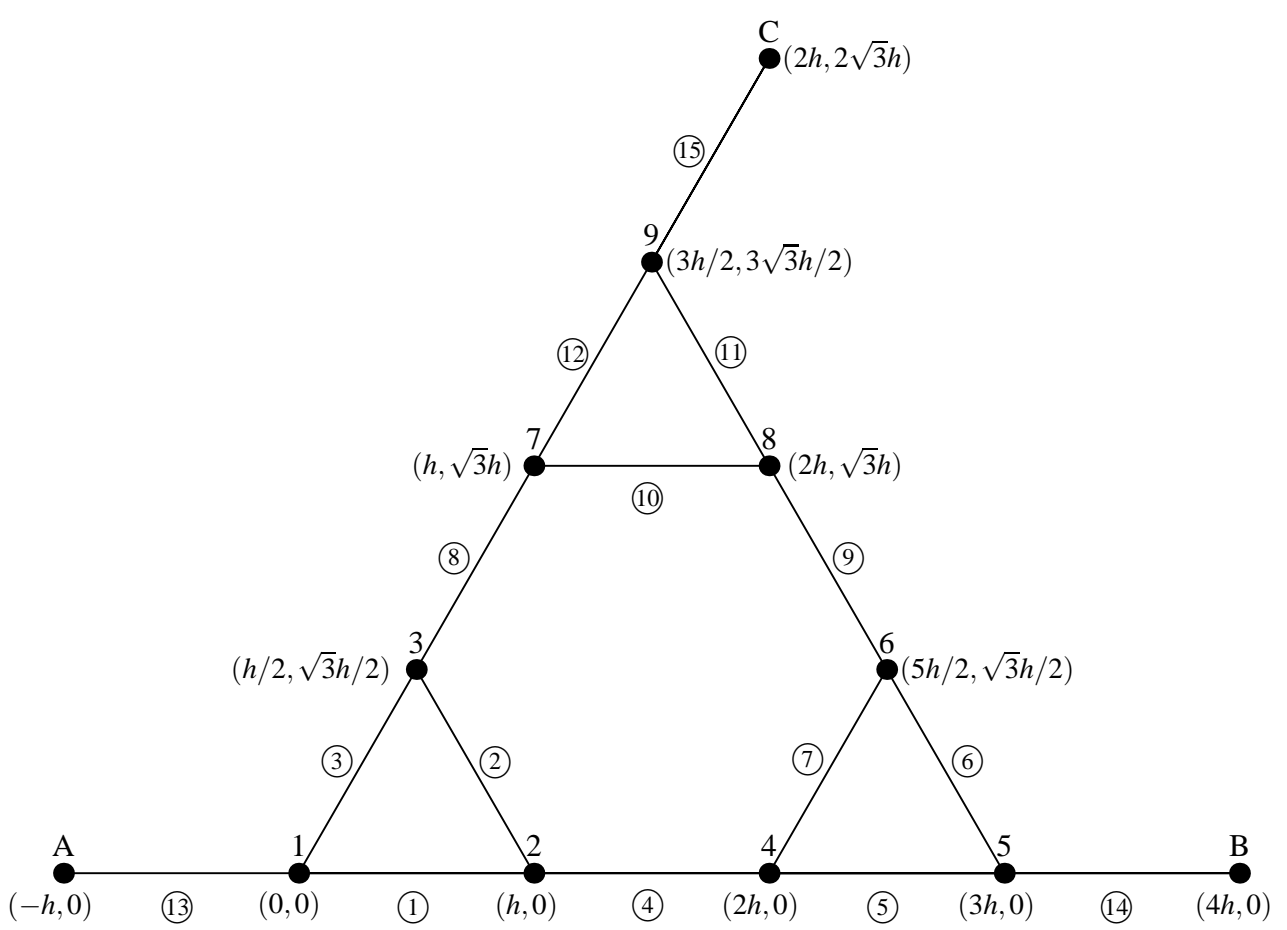

FIG. 4. The Sierpinski Gasket lattice $S G(3)$ at generation level $n=2$. Nodes $A, B$ and $C$ are fictitious nodes used to accommodate the boundary conditions. The lattice has 15 elements (circled numbers), with two vertices adjacent to each element.

Using the isoparametric representation given above

$$
b_{j}^{(n)}= \begin{cases}h \eta_{j}^{(n)} U_{A}, & j=1 \\ h \eta_{j}^{(n)} U_{B}, & j=m \\ h \eta_{j}^{(n)} U_{C}, & j=N \\ 0 & \text { otherwise }\end{cases}
$$

where

$$
\eta_{j}^{(n)}= \begin{cases}\frac{4}{3}-\frac{2}{15} q^{2}, & j=1 \\ 1+\frac{1}{3\left(2^{n+1}-1\right)}+\frac{\left(11-15 \times 2^{n}\right)}{30\left(2^{n+1}-1\right)} q^{2}, & j=m \text { or } N\end{cases}
$$

\subsection{Application of the Mechanical boundary conditions}

Mechanical and electrical loads are now introduced to the transducer at its boundaries. It can be shown that ( Algehyne \& Mulholland (2014))

$$
U_{i}=G_{j i}^{(n)} \bar{\delta}_{j}^{(n)}
$$

where $G_{j i}^{(n)}=\left(\hat{A}_{j i}^{(n)}-\hat{B}_{j i}^{(n)}\right)^{-1}$ represents the Green's transfer matrix. The Green transfer matrix $G_{j i}$ gives the Laplace transform of the displacement at vertex $i$ when vertex $j$ is subject to a unit impulse. 
Here $\hat{A}=A / h$,

$$
\hat{B}_{j i}^{(n)}= \begin{cases}\hat{\gamma}_{j}^{(n)}, & j=i \\ 0 & \text { otherwise, }\end{cases}
$$

$\hat{\gamma}_{j}^{(n)}=\eta_{j}^{(n)} \gamma_{j}$ and $\hat{\delta}_{j}^{(n)}=\eta_{j}^{(n)} \delta_{j}$, where

$$
\gamma_{j}= \begin{cases}\left(1-q \frac{Z_{B}}{Z_{T}}\right)^{-1}, & j=1 \\ \left(1-q \frac{Z_{L}}{Z_{T}}\right)^{-1}, & j=m \text { or } N \\ 0 & \text { otherwise }\end{cases}
$$

and

$$
\delta_{j}= \begin{cases}-\frac{\zeta Q}{\mu_{T} \xi}\left(1-q \frac{Z_{B}}{Z_{T}}\right)^{-1}, & j=1 \\ \left(1-q \frac{Z_{L}}{Z_{T}}\right)^{-1}\left(\frac{\zeta Q}{\mu_{T} \xi}-2 A_{L} q \frac{Z_{L}}{Z_{T}}\right), & j=m \text { or } N \\ 0 & \text { otherwise }\end{cases}
$$

where the mechanical impedance of the load is $Z_{L}=\rho_{L} c_{L} A_{r}$, and of the backing material is $Z_{B}=\rho_{B} c_{B} A_{r}$, and of the transducer is $Z_{T}=\rho_{T} c_{T} A_{r}$, where $\rho_{L}\left(\rho_{B}\right)$ is the density and $c_{L}\left(c_{B}\right)$ is the wave velocity in the load (backing material). Also $\zeta=e_{24} / \varepsilon_{11}$, and $Q$ is the electrical charge applied at one of the transducerelectrical load interfaces. At each generation level of the Sierpinski gasket transducer the ratio of the cross-sectional area of each edge to its length is denoted by $\xi=A_{r} / h$. The overall extent of the lattice $(L)$ is fixed and so the length of the edges will steadily decrease and, by fixing $\xi$, the cross-sectional area will also decrease as the fractal generation level increases (in fact $A_{r}=\xi L /\left(2^{n}-1\right)$ ).

\section{Renormalisation}

From equation (3.18) the desired weightings at each vertex in $\Omega$ is given by

$$
U_{j}^{(n)}=G_{j 1}^{(n)} \hat{\delta}_{1}^{(n)}+G_{j m}^{(n)} \hat{\delta}_{m}^{(n)}+G_{j N}^{(n)} \hat{\delta}_{N}^{(n)} .
$$

It will transpire later that only $U_{1}^{(n)}, U_{m}^{(n)}$ and $U_{N}^{(n)}$ are required and so only the pivotal Green's functions $G_{i j}^{(n)}, i, j \in\{1, m, N\}$ need to be calculated. Temporarily ignoring matrix $\hat{B}$ (this matrix originates from consideration of the boundary conditions) then, due to the symmetries of the $S G(3)$ lattice (and hence in matrix $\left.A^{(n)}\right), \hat{G}_{i i}^{(n)}=\hat{G}_{j j}^{(n)}=\hat{x}$, say, where $i, j \in\{1, m, N\}$ (that is, corner-to-same-corner), and $\hat{G}_{j k}^{(n)}=\hat{G}_{h k}^{(n)}=\hat{y}$, say, where $j, k, h \in\{1, m, N\}, j \neq k \neq h$ (that is, corner-to-other-corner), where

$$
\hat{G}^{(n)}=\left(\hat{A}^{(n)}\right)^{-1} .
$$

At level $n+1$, we denote, $\hat{X}=\hat{G}_{i i}^{(n+1)}$ and $\hat{Y}=\hat{G}_{i j}^{(n+1)}$ where $i, j, \in\{1, m, N\}, i \neq j$. Since $\theta^{(n)}=$ $c_{T} t / h^{(n)}$, then $\mathscr{L}: \theta^{(n)} \rightarrow q^{(n)}$ where $q^{(n)}=i \hat{\omega}^{(n)}=i 2 \pi \hat{f}^{(n)}=i 2 \pi\left(c_{T} / h^{(n)}\right)^{-1} f^{(n)}, \hat{f}^{(n)}$ is the nondimensionalised natural frequency, $\hat{\omega}^{(n)}$ is the nondimensionalised angular frequency and $f^{(n)}$ (and $\omega^{(n)}$ ) are the dimensionalised equivalents. In the renormalisation approach detailed below, $q=q^{(n)}$ is set equal to $q^{(n+1)}$. This simply means that the output from the renormalisation methodology (and hence the electrical impedance and transmission/reception sensitivities) at a given $q$ (fixed) is then that quantity at frequency $f^{(n)}$ at generation level $n$. So when comparing outputs at different generation levels 


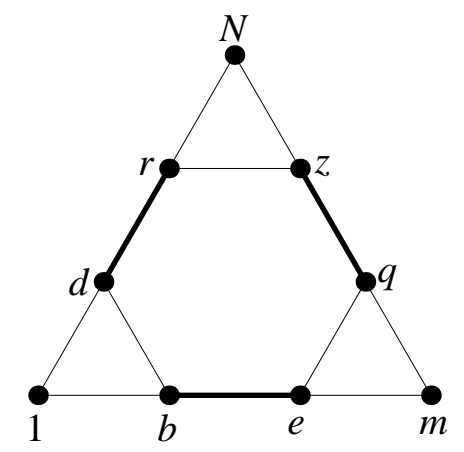

FIG. 5. Three Sierpinski Gasket lattices of generation level $n-1\left(\mathrm{SG}^{(n-1)}(3)\right)$ are connected by the edges in bold $((d, r),(b, e)$ and $(q, z))$ to create the Sierpinski Gasket lattice at generation level $n\left(\mathrm{SG}^{(n)}(3)\right)$.

one must ensure that the frequency is scaled appropriately $\left(\right.$ by $\left.\left(c_{T} / h^{(n)}\right)^{-1}\right)$ when re-dimensionalising. An iterative procedure can be developed from equation (3.6) to give (Algehyne \& Mulholland (2014))

$$
G^{(n)}=\hat{G}^{(n)}+\hat{G}^{(n)} \hat{B}^{(n)} G^{(n)}
$$

The system of linear equation will create the renormalisation recursion relationships for the pivotal Green's functions. Since the subgraphs of Figure 5 only connect to each other at the corners, it will transpire that the recursions in equation (4.3) only involve two pivotal Green's functions, namely, cornerto-corner and corner-to-same-corner; the so called input/output nodes. Solving these (for $\hat{y} \neq 0, \beta \neq 0$ ) gives

$$
\hat{X}=\hat{x}+\frac{2 \beta^{2} \hat{y}^{2}\left(\hat{x}+\beta \hat{x}^{2}-\beta \hat{y}^{2}\right)}{(1+\beta \hat{x}+\beta \hat{y})\left(1-\beta^{2} \hat{x}^{2}-\beta \hat{y}+\beta^{2} \hat{y}^{2}\right)},
$$

and

$$
\hat{Y}=\frac{-\beta \hat{y}^{2}(1+\beta \hat{x}-\beta \hat{y})}{(1+\beta \hat{x}+\beta \hat{y})\left(1-\beta^{2} \hat{x}^{2}-\beta \hat{y}+\beta^{2} \hat{y}^{2}\right)} .
$$

The boundary conditions can now be re-introduced and lead to

$$
\begin{gathered}
x=\frac{\hat{x}+2 \hat{y} \hat{\gamma}_{m} y}{1-\hat{x} \hat{\gamma}_{1}}, \\
y=\frac{\hat{y}}{\left(1-\hat{x} \hat{\gamma}_{1}\right)\left(1-\hat{\gamma}_{m}(\hat{x}+\hat{y})\right)-2 \hat{y}^{2} \hat{\gamma}_{1} \hat{\gamma}_{m}}, \\
z=\frac{\hat{x}+\hat{y} \hat{\gamma}_{1} y+\hat{y} \hat{\gamma}_{m} r}{1-\hat{x} \hat{\gamma}_{m}},
\end{gathered}
$$

and

$$
r=\frac{\hat{y}\left(1+\hat{\gamma}_{1} y\left(1+\hat{\gamma}_{m}(\hat{y}-\hat{x})\right)\right)}{\left(\hat{x} \hat{\gamma}_{m}-1+\hat{y} \hat{\gamma}_{m}\right)\left(\hat{x} \hat{\gamma}_{m}-1-\hat{y} \hat{\gamma}_{m}\right)}
$$

where $x=G_{11}^{(n)}, y=G_{1 N}^{(n)}, z=G_{N N}^{(n)}$ and $r=G_{m N}^{(n)}$ 


\section{Electrical Impedance and Transmission and Reception Sensitivity}

The derivation of the operating characterstics of the device follows similar lines as presented in (Mulholland \& Walker (2011)) and so is omitted (a full derivation is given in (Algehyne \& Mulholland (2014))). The non-dimensionalised electrical impedance is given by

$$
\hat{Z}_{E}(f ; n)=Z_{E} / Z_{0}=\left(\frac{Z_{T}}{C_{0} q \mu_{T} \xi Z_{0}}\right)\left(1+\frac{\zeta^{2} C_{0}}{\mu_{T} \xi}\left(\sigma_{1}+\sigma_{2}\right)\right)
$$

where $\sigma_{1}=\left(1-q\left(Z_{B} / Z_{T}\right)\right)^{-1} \eta_{1}^{(n)}\left(G_{N 1}^{(n)}-G_{11}^{(n)}\right)$ and $\sigma_{2}=\left(1-q\left(Z_{L} / Z_{T}\right)\right)^{-1} \eta_{m}^{(n)}\left(-G_{N m}^{(n)}-G_{N N}^{(n)}+2 G_{1 N}^{(n)}\right)$ and $Z_{0}$ is the series electrical load. The non-dimensionalised transmission sensitivity $\psi$ is given by

$$
\psi(f ; n)=\left(\frac{F}{V}\right) / \zeta C_{0}=\frac{a Z_{L}}{\left(Z_{E}+b\right) \mu_{T} \xi C_{0}} K^{(n)},
$$

where

$$
K^{(n)}=\left(1-q \frac{Z_{L}}{Z_{T}}\right)^{-1}\left(-\eta_{1}^{(n)}\left(1-q \frac{Z_{B}}{Z_{T}}\right)^{-1} G_{m 1}^{(n)}+\eta_{m}^{(n)}\left(1-q \frac{Z_{L}}{Z_{T}}\right)^{-1}\left(G_{m m}^{(n)}+G_{m N}^{(n)}\right)+1\right) .
$$

The non-dimensionalised reception sensitivity $\phi$ is

$$
\begin{aligned}
\phi(f ; n) & =\left(\frac{V}{F}\right)\left(e_{24} L\right) \\
& =\frac{2 \zeta e_{24} L \sigma_{2}}{\xi \mu_{T}}\left(1-\frac{\zeta^{2} a Z_{T}\left(\sigma_{1}+\sigma_{2}\right)}{\left(Z_{E}+b\right) q \mu_{T}^{2} \xi^{2}}-\frac{a Z_{T}}{\left(Z_{E}+b\right) q \mu_{T} \xi C_{0}}\right)^{-1} .
\end{aligned}
$$

Having derived expressions for the main operating characteristics of this new device it is instructive to compare these with a model of a standard device ( Hayward (1984); Hayward et al. (1984)) to assess any practical benefits arising from this novel design.

\section{Numerical Results For Pre-Fractal Ultrasound Transducers}

From a practical perspective, these fractal transducers will only be able to be manufactured at low generation levels. Indeed some preliminary work to manufacture this fractal design at generation level $n=3$ has been undertaken ( Mulholland et al. (2011)). The formulation presented above will allow a comparison of the fractal design with a conventional (Euclidean) design in terms of the key operating characteristics of the reception and transmission sensitivity spectra. Within each, the presence of higher amplitudes, multiple resonances, and improved bandwidth (the range of frequencies over which the performance exceeds a certain decibel level) are the key performance indicators of interest. A careful examination of the transmission and reception sensitivities of the fractal device as the fractal generation level is increased has been performed. However, to keep the presentation here succinct and to produce results that are pertitent to devices that can be physically produced, we will focus on fractal generation level $n=3$. Ultrasonic transducers are resonant devices and their key operating characteristics are governed by the materials they are made of and the length scales used in their designs. The traditional designs have essentially one length scale and hence operate at a single frequency. Assessing the operating ability of a particular transducer is very time consuming. For example, to determine its transmission sensitivity the device has to be immersed in a water tank, input voltages of different frequencies are applied, and a hydrophone placed at some distance from the transducer monitors the output. A simpler 
assessment can be made at an earlier stage by connecting the transducer to an electrical circuit and measuring its electrical impedance over a range of frequencies. A typical profile of this electrical impedance spectrum (magnitude) given by equation (5.1) is shown in Figure 6 (dashed line); it is compared to the equivalent profile given by a model of the traditional design (full line) (Hayward et al. (1984)). The overall trend of the curve is that of a capacitor $(1 / f$ profile) with a series of resonances. The important features of this plot that the design engineer is interested in are the location and magnitude of the first minimum $\left(f_{r}\right)$ and the first maximum $\left(f_{a}\right)$ turning points. The first minimum is where the electrical resonance (or series resonance) occurs and, as this provides the least resistance to the electrical energy being supplied, is the frequency at which the device should be used in transmission mode. This device will produce its maximum force on the mechanical load at this frequency. The absolute value of the electrical impedance at this frequency is also important therefore and the lower it is the higher will be the peak transmission sensitivity of the device. The first maximum (known as the anti-resonance or parallel resonance frequency) is where the mechanical resonance of the device peaks and is therefore the optimal frequency to operate the device in reception mode. As can be seen in Figure 6 the resonant behaviour of the traditional design is quite simple with a series of periodic minimum/maximum pairs. Note that we have not accounted for damping in our model and so the higher frequency turning points are far more pronounced than would be observed experimentally. It can be seen that for the traditional design $f_{r}=0.9 \mathrm{MHz}$ and $\left|Z_{E}\left(f_{r} ; 3\right)\right|=26 \mathrm{~dB}$ and $f_{a}=1.2 \mathrm{MHz}$. From the parameter values for PZT5-H (see (Auld (1973))) then the piezoelectrically stiffened velocity $\left(c_{T}\right)$ in equation (2.9) is approximately $2370 \mathrm{~m} / \mathrm{s}$ and, with an overall device length of $L=1 \mathrm{~mm}$, then the first mechanical resonant frequency is approximately $f_{a}=c_{T} /(2 L)=1.2 \mathrm{MHz}$. As discussed above, these frequencies correspond precisely to the first maximum in the transmission sensitivity plot (Figure 7, full line) at 09.MHz, and the reception sensitivity plot (Figure 8, full line) at 1.2MHz. For the Sierpinski gasket design the electrical impedance resonance frequencies are much higher $\left(f_{r}=2.2 \mathrm{MHz}\right.$ and $\left.f_{a}=2.3 \mathrm{MHz}\right)$ and this suggests that it is a complex interaction between the edge lengths in the graph associated with the various generation levels that are causing these resonances; so the internal geometry is dictating the device behaviour as anticipated. Importantly, the magnitude of the electrical impedance at the electrical resonance frequency is lower than the traditional design; there is about a $2 \mathrm{~dB}$ drop. This results in the transmission sensitivity spectrum having a much larger gain; there is a $15 \mathrm{~dB}$ improvement from a $28 \mathrm{~dB}$ transmission sensitivity gain in the traditional design to around $43 \mathrm{~dB}$ in this fractal design. However, this peak in the transmission sensitivity results in a reduced bandwidth; if we take the noise floor to be $3 \mathrm{~dB}$ below the peak gain of the traditional design (that is $25 \mathrm{~dB}$ ) then the operational bandwidth of the traditional design is $0.25 \mathrm{MHz}$ (or $28 \%$ ) whereas the fractal design only has an operational bandwidth of around $0.07 \mathrm{MHz}$ (or 3\%). It should be borne in mind of course that no matching layers (or indeed an optimised backing layer) have been used in this design, and that the transducer is solely composed of the piezoelectric material. In practical designs the piezoelectric material is combined with a low mechanical impedance polymer to result in a composite material that more closely matches the mechanical impedance of the load material. This leads to a reduction in the interface reflection coefficient and hence more energy is transferred (and received from) into the object that the transducer is inspecting. So this accounts in part for the low values of these bandwidths. The reception sensitivity tells a similar story with the fractal transducer having a peak gain of around $25 \mathrm{~dB}$ which is around $10 \mathrm{~dB}$ higher than the standard design. Again, if we take the noise floor to be around $3 \mathrm{~dB}$ lower than the peak gain of the standard design (so a $12 \mathrm{~dB}$ level) then the operational bandwidth of the fractal design is $0.11 \mathrm{MHz}(0.05 \%)$ compared to $0.19 \mathrm{MHz}(0.16 \%)$ from the standard design. 


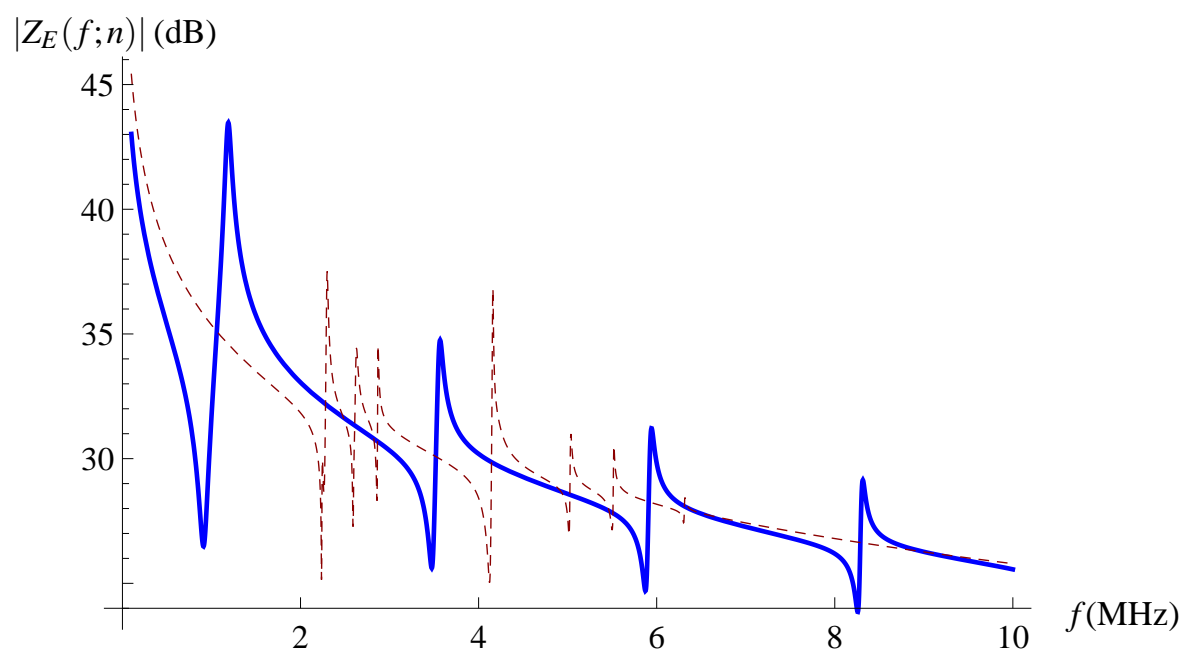

FIG. 6. Non-dimensionalised electrical impedance (equation (5.1)) versus frequency for the $S G(3)$ lattice transducer at fractal generation level $n=3$ (dashed line). The non-dimensionalised electrical impedance of the standard (Euclidean) transducer is plotted for comparison (full line). Parameter values are given in (Auld (1973)) for PZT-5H.

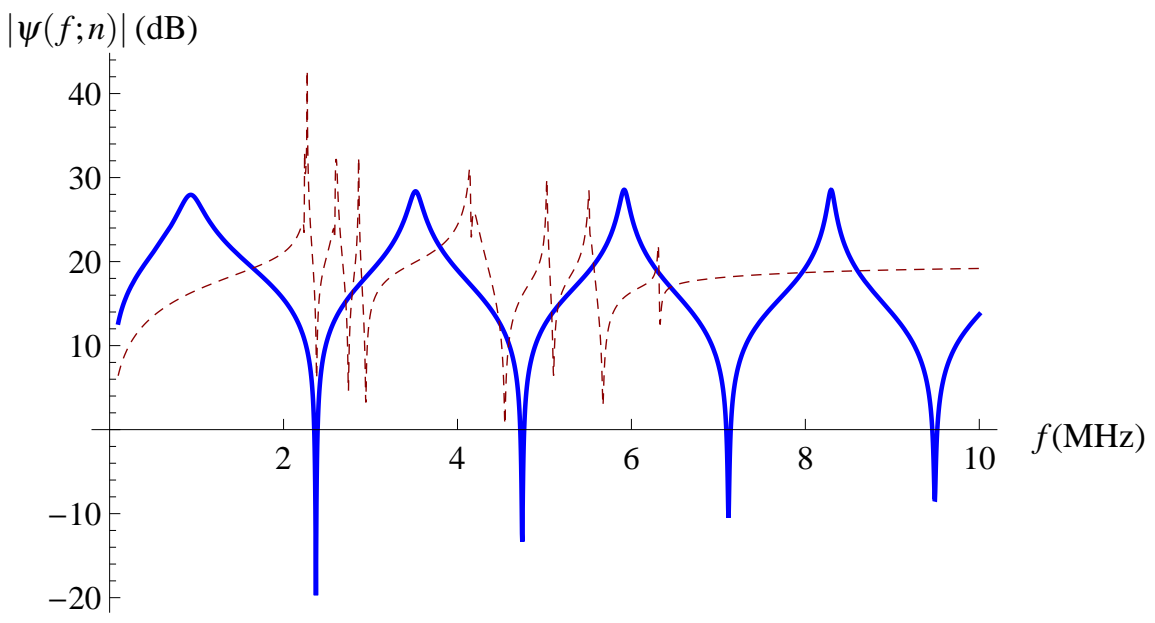

FIG. 7. Non-dimensionalised transmission sensitivity (equation (5.2)) versus frequency for the $S G(3)$ lattice transducer at fractal generation level $n=3$ (dashed line). The non-dimensionalised transmission sensitivity of the standard (Euclidean) transducer is plotted for comparison (full line). Parameter values are given in (Auld (1973)) for PZT-5H. 


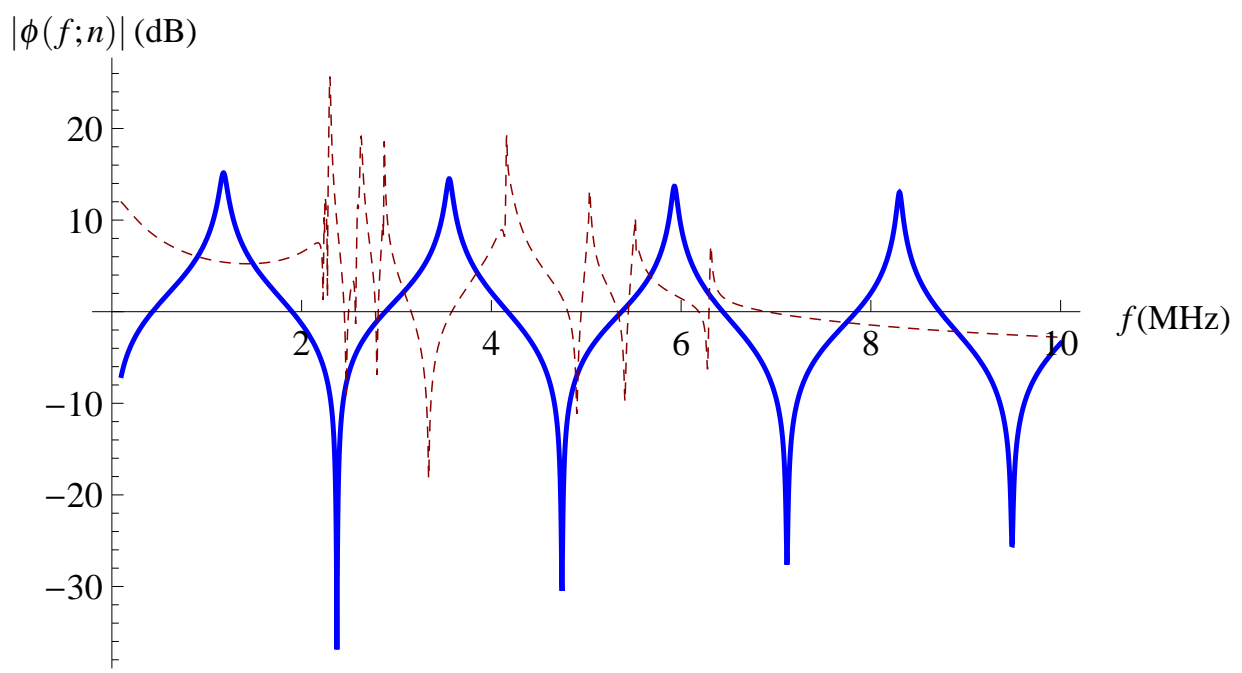

FIG. 8. Non-dimensionalised reception sensitivity (equation (5.4)) versus frequency for the $S G(3)$ lattice transducer at fractal generation level $n=3$ (dashed line). The non-dimensionalised reception sensitivity of the standard (Euclidean) transducer is plotted for comparison (full line). Parameter values are given in (Auld (1973)) for PZT-5H.

The convergence of the device's operating characteristics as the fractal generation level increases can be examined. To do this the norm of the difference between the energy in the power spectrum at successive generation levels, integrated with respect to frequency, is calculated for the transmission/reception sensitivities, as follows

$$
\sum_{i=1}^{m}\left|\psi\left(f_{i} ; n\right)-\psi\left(f_{i} ; n+1\right)\right|=\psi^{*}(n)
$$

and

$$
\sum_{i=1}^{m}\left|\phi\left(f_{i} ; n\right)-\phi\left(f_{i} ; n+1\right)\right|=\phi^{*}(n)
$$

Figure 9 shows the dependence of these norms on the generation level and it can be seen that both sensitivities converge by a relatively low generation level. Scrutiny of the underlying spectra shows that the transmission sensitivity accrues more and more resonances as the fractal generation $n$ increases. As the length scale of the smallest edge is decreasing with $n$ then resonances at higher frequencies appear; again the lack of damping in the model permits these resonances to have amplitudes which would not be present in an experimental setting. This accounts for the initial upward trend observed in Figure 9. As $n$ is increased further, then the various peaks become quite dense and a very flat response emerges which doesnt change over the frequency range of interest (up to $10 \mathrm{MHz}$ ). Hence, the successive spectra start to reach a steady state and this accounts for the downward trend and eventual steady state that is reached after $n=10$. A similar story holds for the reception sensitivity but here there is still a fluctuation in the value of the flat response as $n$ increases. This at first gives an initial rise in the differences between the energy in successive spectra $(n=5,6,7)$ but then as $n$ is increased further this difference decreases until a steady state is achieved around $n=15$. 


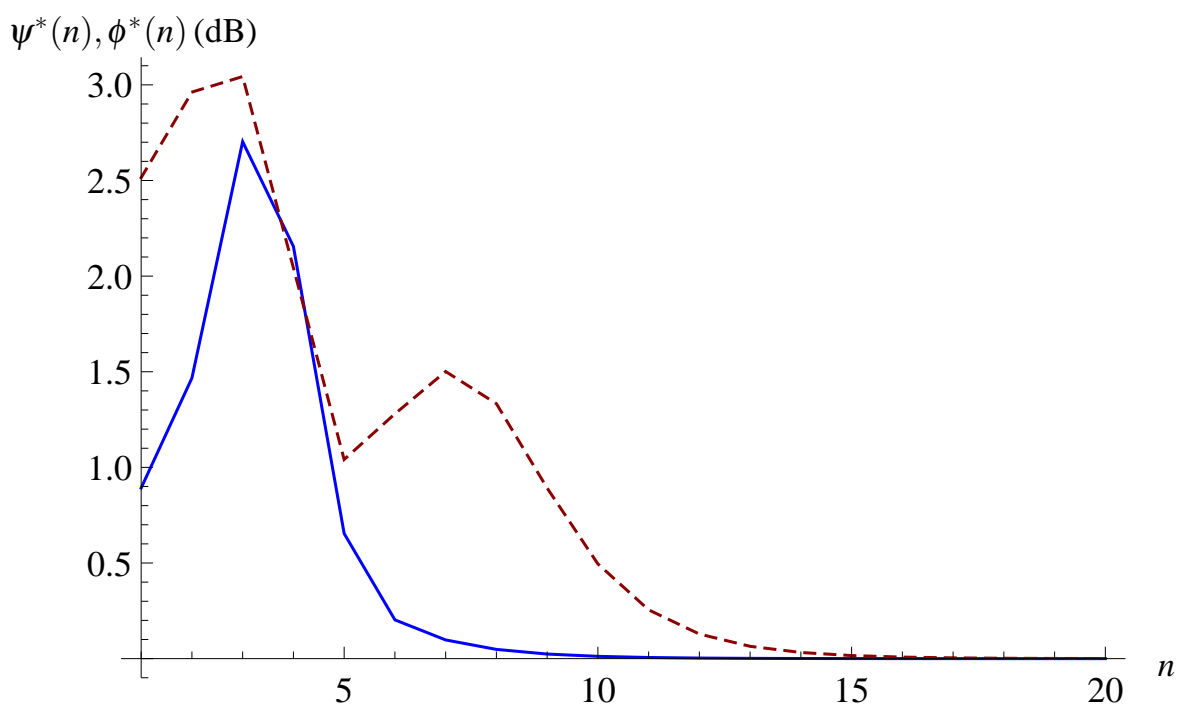

FIG. 9. The convergence of the transmission and reception sensitivities is examined by plotting the differences in the energies in successive spectra as the fractal generation level increases. Non-dimensionalised transmission sensitivity $\left(\psi^{*}(n)\right)$ (equation $(6.1)$ ) (full line) and non-dimensionalised reception sensitivity $\left(\phi^{*}(n)\right)$ (equation (6.2)) (dashed line) versus the fractal generation level. The transmission sensitivity converges by generation level $n=10$ and the reception sensitivity by generation level $n=16$, over this frequency range where $f_{i} \in[0.1,10] \mathrm{MHz}$

\begin{tabular}{lccc}
\hline Design Parameter & Symbol & Magnitude & Dimensions \\
\hline Parallel electrical impedance load & $Z_{P}$ & 1000 & Ohms \\
Series electrical impedance load & $Z_{0}$ & 50 & Ohms \\
Length of transducer & $L$ & 1 & $\mathrm{~mm}$ \\
Mechanical impedance of the front load & $Z_{L}$ & 1.5 & MRayls \\
Mechanical impedance of the backing layer & $Z_{B}$ & 2 & MRayls \\
Wave speed in the front load & $c_{L}$ & 1500 & $\mathrm{~ms}^{-1}$ \\
Wave speed in the backing layer & $c_{B}$ & 1666 & $\mathrm{~ms}^{-1}$ \\
Density of the front load & $\rho_{L}$ & 1000 & $\mathrm{kgm}^{-3}$ \\
Density of the backing layer & $\rho_{B}$ & 1200 & $\mathrm{kgm}^{-3}$ \\
\hline
\end{tabular}

Table 1. Parameter Values for the Sierpinski Gasket Transducer ( Mulholland et al. (2007); Mulholland \& Walker (2011)). For PZT-5H parameters see (Auld (1973))

\section{Conclusions}

A model of a piezoelectric ultrasound transducer with a fractal geometry has been constructed and its operational qualities compared with that of a standard (Euclidean) design. The fractal that was used to simulate this self-similar transducer was the Sierpinski gasket ( Falconer \& Hu (2001)). The lattice counterpart of the Sierpinski gasket SG(3) ( Schwalm (1988); Mulholland (2008)) was used to express 
the electrical and mechanical fields in terms of a finite element methodology. This paper is the first time that a finite element analysis has been performed on this structure and this necessitated the introduction of some new basis functions. The fractal design has multiple length scales (the standard design typically has a single length scale) and, since these are resonating devices, this resulted in a rich set of resonating frequencies. Indeed the broadband resonators found in nature and in musical instruments rely on this principle. The finite element formulation resulted in a matrix equation whose solution yielded to a renormalisation approach. This is turn led to a small set of recursion relationships for the pivotal Green's functions that drive the calculation of the transmission/reception sensitivities of the device. The focus was on low generation levels of the fractal as these are most likely to adhere to manufacturing constraints. The results showed that the fractal transducer resonates at many more frequencies than the standard (Euclidean) transducer. Importantly, at certain generation levels the fractal transducer gave rise to a significantly higher amplitude transmission and reception sensitivity than the standard (Euclidean) design. The convergence of the fractal device's performance as the fractal generation level increases was also considered. It was seen that, in both transmission and reception modes, the outputs converge by generation levels $n=10$ and $n=16$ respectively. As this paper considers the out-of-plane motion of the vertices in the fractal design then the resonant modes correspond to the flexural behaviour of the device (transverse waves). This in contrast to the work in (Mulholland \& Walker (2011)) where only the in-plane vibrations were calculated; so only longitudinal waves modes are considered. The major advantage in the renormalisation approach is that the operational characteristics of the device can be calculated in a fraction of a second even for large fractal generation levels. For example, on a standard desktop PC, with the model implemented in Mathematica, the calculation of the transmission sensitivity spectrum at fractal generation level $n=9$ took $0.45 \mathrm{~s}$. The finite element approach would also provide a natural framework for an error analysis to be conducted and an assessment of role of the polynomial degree of the basis functions on this error investigated for example. This will be the subject of our future investigations. These encouraging results suggest that it will be worthwhile studying other fractal designs. There are other fractal designs that will yield to a similar analysis; these include the Lindstrom snowflake (Derfel et al. (2012)), the Vicsek set, the rotated triangle set (Barlow (1998)), the ARC(p) family of graphs and the Hulfer-Blumen fractal (Giona et al. (1996)). A program to manufacture these fractal transducers has been instigated and the comparison between the theoretical and experimental results will be the subject of a future investigation. The model could be improved in a number of ways; the spatial degrees of freedom of each vertex could be extended to allow movement in more than one direction and this would lead to a vector elastodynamic model, the electric field is homogenized over the lattice in the current model (this is the quasi-static approximation which uses the separation of time scales between the electrical and mechanical waves) and this could be recast to involve the time dependent Maxwell equations, higher order polynomials could be used for the underlying basis functions, damping could be included to account for frequency dependent losses, mechanical loads could be applied across the face of the lattice to facilitate an investigation of the transducer directivity patterns. One weakness in the approach is that it can only be applied to post-critically finite (finitely ramified) fractals; these are graphs that have a finite number of bridges. The method very much relies on the use of an initial (generator) shape being rescaled and multiple copies of this shape being connected together at the next generation level. The initial shape could have a range of length scales within it but the method would require strict adherence to the same scale factor being applied to this shape at each fractal generation level. As mentioned earlier it is not possible to compare the results here to the previously conducted finite difference calculation as this studied a different mode of operation of the device. However, it would of course be straightforward to conduct such a comparison by recasting the parameterisation in this paper so that longitudinal waves could be simulated. 


\section{Acknowledgements}

The authors gratefully acknowledge the support given by the Ministry of Higher Education, University of Tabuk in Saudi Arabia and the Royal Embassy of Saudi Arabia in UK.

\section{References}

Abdulbake, J., Mulholland, A. J. \& Gomatam, J. (2004) Existence and Stability of ReactionDiffusion Waves on a Fractal Lattice, Chaos, Solitons and Fractals, 20(4), 799-814.

Abdulbake, J., Mulholland, A. J.\& Gomatam, J. (2003) A Renormalization Approach To Reaction-Diffusion Processes On Fractals, Fractals, 11(4), 315-330.

Algehyne, E. A. \& Mulholland, A. J. (2014) A Finite Element Approach to Modelling Fractal Ultrasonic Transducers, Department of Mathematics and Statistics, University of Strathclyde, Depatmental report, $\mathbf{3 5}(1)$.

Auld, B. A. (1973) Acoustic Fields and Waves in Solids, New York, John Wiley and Sons, 1.

BARLOW, M. T. (1998) Diffusion on fractals, Lectures on Probability Theory and Statistics, Berlin: Springer, ed P Bernard, (Lecture Notes in Mathematics vol (1690)), 1-121.

Chiselev, A. M., Moraru, L. \& Gogu, A. (2009) Localization of an object using a bat model inspired from biology, Romanian, J. Biophys, 19(4), 251-258.

DERFEL, G., GRABNER, P. J. \& VOGL, F. (2012) Laplace operators on fractals and related functional equations, J. Phys. A: Math. Theor., 45(463001), doi:10.1088/1751-8113/45/46/463001.

Eberl, D. F., Hardy, R. W. \& Kernan, M. J. (2000) Genetically similar transduction mechanisms for touch and hearing in Drosophila, J. Neurosci, 20(16), 5981-5988.

FAlconer, K. (2003) Fractal Geometry: Mathematical Foundations and Applications, Chichester, England John Wiley and Sons Ltd,

FAlconer, K. \& HU, J. (2001) Nonlinear Diffusion Equations on Unbounded Fractal Domains, $J$ Math Anal App, 256, 606-624.

GionA, M. (1996) Transport Phenomena in Fractal and Heterogeneous Media: Input/Output Renormalization and Exact Results Chaos, Solitons and Fractas, 7(9), 1371-1396.

GIONA, M., SCHWALM, W.A., SCHWALM, M.K. \& ADROVER, A. (1996) Exact solution of linear transport equations in fractal media - I. Renormalization analysis and general theory, Chem. Eng. Sci, 51(20), 4717-4729.

HAYWARD, G. (1984) A systems feedback representation of piezoelectric transducer operational impedance, Ultrasonics, 22, 153-162.

Hayward, G., McLeod, C. J. \& Duranni, T. S. (1984) A systems model of the thickness mode piezoelectric transducer, JASA, 76(2), 369-382.

Kigami, J. (2001) Analysis on Fractals, Cambridge, UK, Cambridge University Press, . 
MiLes, R. N.\& HoY, R. R. (2006) The development of a biologically-inspired directional microphone for hearing aids, Audiol Neurootol, 11(2), 86-94.

Montero de Espinosa, F., Martinez, O., Segura, L.E. \& Gomez-Ullate, L. (2005) Double frequency piezoelectric transducer design for harmonic imaging purposes in NDT, IEEE. TUFFC, 52(6), 980-986.

Mulholland, A. J. (2008) Bounds on the Hausdorff dimension of a renormalisation map arising from an excitable reaction-diffusion system on a fractal lattice, Chaos, Solitons and Fractals, 35(2), 274-284.

Mulholland, A. J. \& Walker, A. J. (2011) Piezoelectric Ultrasonic Transducers With Fractal Geometry, Fractals, 19(4), 469-479.

Mulholland, A. J., Ramadas, N., O'Leary, L., Parr, A.C. S., Troge, A., Hayward, G. \& Pethrick, R. A. (2007) A Theoretical Analysis of a Piezoelectric Ultrasound Device with an Active Matching Layer, Ultrasonics, 47(1), 102-110.

Mulholland, A. J., Walker, A. J., Mackersie, J. W., O'Leary, R. L., Gachagan, A. \& Ramadas, N. (2011) The Use of Fractal Geometry in the Design of Piezoelectric Ultrasonic Transducers, Ultrasonics Symposium (IUS), 2011 IEEE International, 1559-1562.

MÜLLER, R. (2004) A numerical study of the role of the tragus in the big brown bat, JASA, 116(6), $3701-3712$.

MÜller, R., Lu, H., Zhang, S. \& Peremans, H. (2006) A helical biosonar scanning pattern in the chinese noctule Nycatalus plancyi, JASA, 119(6), 4083-4092.

Nadrowski, B., Albert, J. T. \& Göpfert, M. C. (2008) Transducer-Based Force Generation Explains Active Process in Drosophilia Hearing, Curr. Biol, 18, 1365-1372.

Orr, L. A., Mulholland, A. J., O’leary, R. L., Parr, A., Pethrick, R. \& Hayward, G. (2007) Theoretical modelling of frequency dependent elastic loss in composite piezoelectric transducers, Ultrasonics, 47(1), 130-137.

Robert, D.\& GöPfERT, M. C. (2002) Novel schemes for hearing and orientation in insects, Curr. Opin. Neurobiol, 12, 715-720.

Schwalm, W. A. \& Schwalm, M. K. (1988) Extension theory for lattice Green functions, Phys.Rev.B, 37(16), 9524-9542.

YANG, J. (2006) The Mechanics of Piezoelectric Structures, Singapore, World Scientific,.

YANG, J. (2006) Analysis of Piezoelectric Devices, Singapore, World Scientific,.

\section{Nomenclature}

The tables below provide a full nomenclature of terms used within the article. It is worth noting that, as far as notation concerned, the literature is not consistent and care should be taken when comparing with other work. 


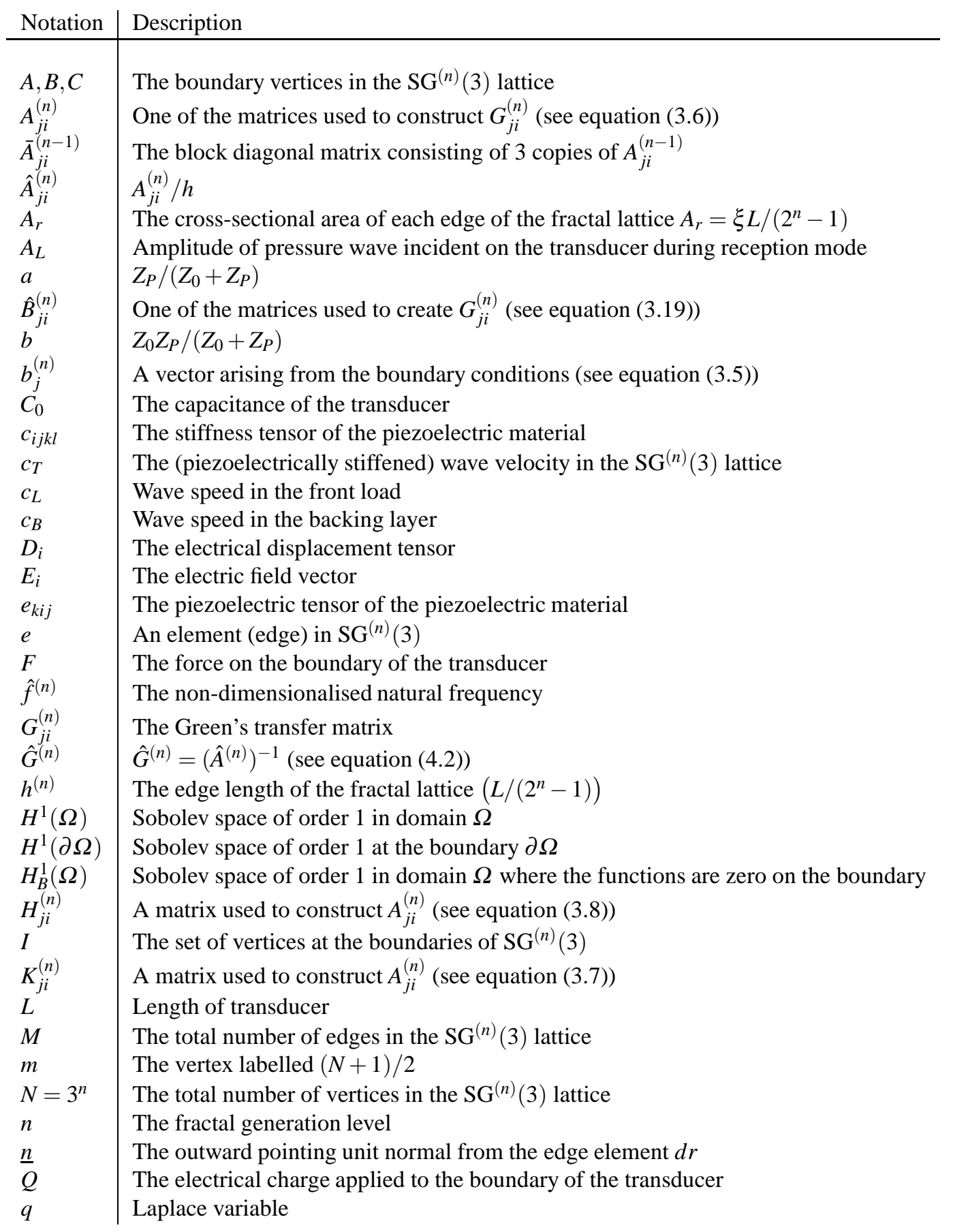




\begin{tabular}{|c|c|}
\hline Notation & Description \\
\hline$S G^{(n)}(3)$ & The Sierpinski gasket lattice of degree 3 \\
\hline$S_{k l}$ & The strain tensor \\
\hline$S$ & The finite dimensional subspace correspondury to $H^{1}(\Omega)$ \\
\hline$S_{B}$ & The finite dimensional subspace correspondury to $H_{B}^{1}(\Omega)$ \\
\hline$s$ & The parameter used in the isoparametric description of each element \\
\hline$T_{i j}$ & The stress tensor \\
\hline$t$ & Time \\
\hline$u_{i, j}$ & The displacement gradients \\
\hline$u_{i}$ & The component of displacement in the direction of the $i^{\text {th }}$ basis vector \\
\hline$u_{\partial \Omega}$ & The displacement in the boundary of $\mathrm{SG}^{(n)}(3)$ \\
\hline$U_{B}$ & The function that approximates the displacement at the boundary \\
\hline$U$ & The approxmate displacement in region $\Omega$ (see equation (3.3) \\
\hline$U_{B_{i}}$ & The displacement at the boundary vertex $B_{i}$ \\
\hline$U_{A}, U_{B}, U_{C}$ & The displacement of the boundary vertices $\{A, B, C\}$ \\
\hline$V_{j i}^{(n)}$ & $\begin{array}{l}\text { The adjacency matrix for the subgraph of } \mathrm{SG}^{(n)}(3) \text { consisting of the edges } \\
\text { that connect each of the three } \mathrm{SG}^{(n-1)}(3) \text { graphs }\end{array}$ \\
\hline$V$ & The voltage applied to the transducer \\
\hline$W$ & The test function in the finite dimensional space $S_{B}, W=\phi_{j}$ \\
\hline$w$ & The test function in the infinite dimensional weak formulation \\
\hline$r$ & $r=G_{m N}^{(n)}$ \\
\hline$\hat{X}$ & $\hat{X}=\hat{G}_{i i}^{(n+1)}$ where $i \in\{1, m, N\}$ \\
\hline$\underline{x}$ & The spatial coordinates (cartesian) \\
\hline$\underline{x}_{j}$ & The spatial location of vertex $j$ in the $\mathrm{SG}^{(n)}(3)$ lattice \\
\hline$x$ & $x=G_{11}^{(n)}$ \\
\hline$\hat{x}$ & $\hat{x}=\hat{G}_{i i}^{(n)}=\hat{G}_{j j}^{(n)}$ where $i, j \in\{1, m, N\}$ \\
\hline$\hat{Y}$ & $\hat{Y}=\hat{G}_{i j}^{(n+1)}$ where $i, j, \in\{1, m, N\}, i \neq j$ \\
\hline$y$ & $y=G_{1 m}^{(n)}=G_{1 N}^{(n)}$ \\
\hline$\hat{y}$ & $\hat{y}=\hat{G}_{i k}^{(n)}=\hat{G}_{h k}^{(n)}$ where $j, k, h \in\{1, m, N\}, j \neq k \neq h$ \\
\hline$Z_{B}$ & Mechanical impedance of backing layer \\
\hline$Z_{L}$ & Mechanical impedance of the front load \\
\hline$Z_{T}$ & Mechanical impedance of the transducer \\
\hline$Z_{P}$ & Parallel electrical impedance load \\
\hline & Series electrical impedance load \\
\hline$\hat{Z}_{E}(f ; n)$ & The non-dimensionalised electrical impedance of the $\mathrm{SG}^{(n)}(3)$ lattice \\
\hline$z$ & $z=G_{m m}^{(n)}=G_{N N}^{(n)}$ \\
\hline
\end{tabular}




\begin{tabular}{l|l} 
Notation & Description \\
\hline & Non-dimensionalised parameter given by equation (3.14) \\
$\beta$ & Non-dimensionalised parameter given by equation (3.14) \\
$\gamma_{j}$ & Non-dimensionalised parameter given by equation (3.20) \\
$\hat{\gamma}_{j}^{(n)}$ & $\eta_{j}^{(n)} \gamma_{j}$ \\
$\delta_{j}$ & Non-dimensionalised parameter given by equation (3.21) \\
$\hat{\delta}_{j}^{(n)}$ & $\eta_{j}^{(n)} \delta_{j}$ \\
$\varepsilon_{i k}$ & The permittivity tensor \\
$\zeta$ & $e_{24} / \varepsilon_{11}$ \\
$\eta_{j}^{(n)}$ & Non-dimensionalised parameter given by equation (3.17) \\
$\theta$ & The non-dimensionalised temporal variable \\
$\mu_{T}$ & The (piezoelectrically stiffened) shear modulus \\
$\xi$ & $A_{r} / h$ \\
$\rho_{B}$ & Density of the backing layer \\
$\rho_{L}$ & Density of the front load \\
$\rho_{T}$ & The density of the piezoelectric material \\
$\phi_{j}$ & The localised basis function at vertex $j$ \\
$\phi(f ; n)$ & The non-dimensionalised reception sensitivity \\
$\phi^{*}(n)$ & The reception sensitivity integrated over all frequencies \\
$\psi(f ; n)$ & The non-dimensionalised transmission sensitivity \\
$\psi^{*}(n)$ & The transmission sensitivity integrated over all frequencies \\
$\Omega$ & The set of points lying on the edges and vertices of SG ${ }^{(n)}(3)$ \\
$\partial \Omega$ & The region's boundary \\
$\hat{\omega}^{(n)}$ & The nondimensionalised angular frequency \\
&
\end{tabular}

